\title{
Valley Physics in Non-Hermitian Artificial Acoustic Boron Nitride
}

\author{
Mudi Wang, ${ }^{1}$ Liping Ye, ${ }^{1}$ J. Christensen,,${ }^{2, *}$ and Zhengyou Liu ${ }^{1,3, \dagger}$ \\ ${ }^{1}$ Key Laboratory of Artificial Micro- and Nano-structures of Ministry of Education \\ and School of Physics and Technology, Wuhan University, Wuhan 430072, China \\ ${ }^{2}$ Department of Physics, Universidad Carlos III de Madrid, Avenida de la Universidad 30, 28916 Leganes (Madrid), Spain \\ ${ }^{3}$ Institute for Advanced Studies, Wuhan University, Wuhan 430072, China
}

(Received 19 October 2017; published 12 June 2018)

\begin{abstract}
The valley can serve as a new degree of freedom in the manipulation of particles or waves in condensed matter physics, whereas systems containing combinations of gain and loss elements constitute rich building units that can mimic non-Hermitian properties. By introducing gain and loss in artificial acoustic boron nitride, we show that the acoustic valley states and the valley-projected edge states display exotic behaviors in that they sustain either attenuated or amplified wave propagation. Our findings show how non-Hermiticity introduces a mechanism in tuning topological protected valley transports, which may have significance in advanced wave control for sensing and communication applications.
\end{abstract}

DOI: 10.1103/PhysRevLett.120.246601

The discrete valley degree of freedom, which can be viewed as pseudospin, attracts a lot of attention in the community of condensed matter physics. Thanks to the large separation between the valleys in momentum space, the intervalley scattering occurs scarcely, thus providing a solid candidate for a new type of information carrier and processor. Many exciting phenomena, such as valley polarized currents [1-9], valley filters [10-13], and valley Hall effects [14-18] have been theoretically predicted and experimentally observed. Moreover, bulk valley transport, such as the notable vortex nature of eigenstates [19], valley-selective excitation [20], and chirality-locked beam splitting behavior [21], have also been unveiled. Meanwhile, the topological valley-projected edge states (VPESs), which are immune to lattice imperfections, have also been extensively reported in optics [22-31] and acoustics systems [32-38].

Parity-time $(P T)$ symmetric structures [39-42] are systems composed of complex potentials, which are neither parity $(P)$ symmetric nor time $(T)$ reversal symmetric alone but are symmetric after the combined operations of $P$ inversion and $T$ reversal. The eigenvalues of the $P T$ symmetric Hamiltonian can be found to be real, despite the non-Hermitian nature of the system. However, when entering the $P T$ broken phase, many intriguing approaches have laid the foundation to realize one-way propagation of optical waves [43-49] or acoustic waves [50-55]. In particular, recent experiments comprising non-Hermitian acoustic structures presented a new route for sound wave manipulation by cleverly combing gain and loss units [52-55].

In this Letter, we introduce gain and loss into phononic crystals that sustain acoustic valleys where the aim is to investigate the effect of non-Hermiticity on the valley transport. In order to design such a non-Hermitian phononic crystal, we engineer artificial acoustic boron nitride $(\mathrm{ABN})$ with spatially alternating gain and loss located, respectively, at two sublattices. In the realization of $A B N$, gain or loss is modeled by introducing complex mass densities. The non-Hermitian ingredients are provided by setting up a complex mass density, which has been shown to be feasible in practice $[50,52]$. However, we like to stress that engineering non-Hermitian acoustic properties is possible also by utilizing a complex bulk modulus in active practical systems [56]. The complex band structures and the propagation of the obstacle-immune edge states have all been computed with COMSOL Multiphysics.

Artificial ABN possesses a hexagonal lattice structure consisting of fluid cylinders $A$ (black) and $B$ (white) placed, respectively, at the two sublattices in air, as illustrated in Fig. 1(a) [58]. The acoustic parameters for air are the bulk modulus $\kappa_{0}=1.4 \times 10^{5} \mathrm{~Pa}$ and the mass density $\rho_{0}=$ $1.21 \mathrm{~kg} / \mathrm{m}^{3}$. For cylinder $A$, which has a radius of $r_{A}=$ $0.35 a_{0}\left(a_{0}\right.$ is the nearest distance between $A$ and $\left.B\right)$, the bulk modulus $\kappa_{A}=0.135 \kappa_{0}$, and the mass density $\rho_{A}=$ $(1-\gamma i) \rho_{0}$, where $\gamma$ is the controlling factor describing the gain (when negative) or loss (when positive) in $A$; For cylinder $B, \quad r_{B}=0.30 a_{0}, \quad \kappa_{B}=0.135 \kappa_{0}, \quad$ and $\rho_{B}=$ $(1+n \gamma i) \rho_{0}$, with $n \gamma$ controlling the amount of gain or loss in $B$. Throughout this Letter, $n=1.4$ is taken. Since $n$ is larger than 1 , while the cylinder radius of $B$ is smaller than in $A$, the gain and loss in $A$ and $B$ are approximately balanced. The lattice constant of the ABN structure is $a=\sqrt{3} a_{0}$.

Given, for example, $\gamma=0.01$, we calculate the complex band structure of ABN as shown in Fig. 1(b). For the real part of the complex band structure, the solid lines, we can see that there is an omnidirectional band gap between the 
(a)
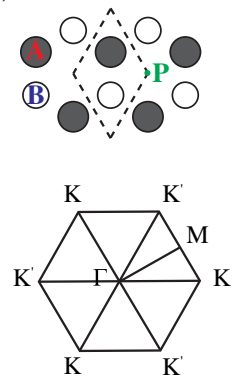

(b)

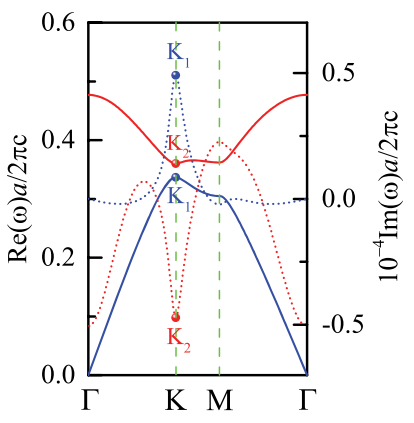

(c)

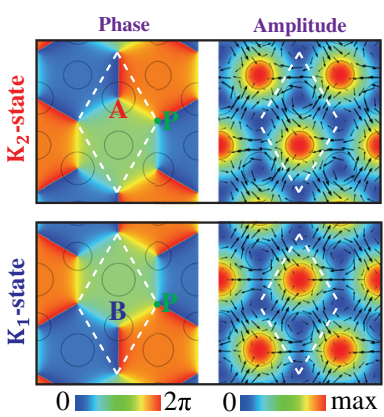

FIG. 1. (a) Top panel, schematic of artificial ABN consisting of cylinders $A$ and $B$ in air, $P$ is indicating the position with $C_{3}$ symmetry. Bottom panel: the corresponding FBZ. (b) The complex band structure of ABN with gain and loss factor $\gamma=0.01$. The real part (solid line) and imaginary part (dash line) of the frequency are plotted separately. The red lines represent the upper band, while the blue lines represent the lower band. (c) Phase and amplitude distributions for valley states $K_{1}$ and $K_{2}$. The arrows in the right panels represent the corresponding time averaged Poynting vectors.

first and second bands, where the extrema, located at $K$ (i.e., $K_{1}$ and $K_{2}$ ) and $K^{\prime}$ (not shown), are called valleys. From the imaginary part, the dashed lines, we can see that the valley state at $K$ (or $K^{\prime}$ ) has maximal attenuation or amplification as the imaginary part is extremal at $K$ (or $K^{\prime}$ ). Specifically, the valley state $K_{1}$ at the first band is an attenuating state as the imaginary frequency is positive while the valley state $K_{2}$ at the second band is an amplifying state as the imaginary frequency is negative. The properties of the valley states $K_{1}^{\prime}$ and $K_{2}^{\prime}$ at $K^{\prime}$ can be deduced similarly.

Figure 1(c) shows the valley states $K_{1}$ (bottom panels) and $K_{2}$ (top panels), in which the left panels illustrate the computed phase $\phi(\boldsymbol{r})$, whereas the right panels represent the amplitude $|p(\boldsymbol{r})|$. At point $P$ (with threefold rotational symmetry $C_{3}$ ), the pressure amplitudes vanish and the phases become singular. Surrounding $P$, there are flux vortices in both $K_{1}$ and $K_{2}$ states; however, the vortex is anticlockwise in the $K_{1}$ state (with charge of +1 ) but clockwise in the $K_{2}$ state (with charge of -1). As can be seen, for the $K_{1}$ state, the amplitude maximum is inside cylinder $A$, which is a loss medium, while for the $K_{2}$ state the amplitude maximum is inside cylinder $B$, which is a gain medium, thus explaining why $K_{1}$ is an attenuating state and $K_{2}$ is an amplifying state.

As the $K_{1}$ or $K_{2}$ state carry a valley vortex with charge +1 or -1 , we can use a chiral point source with topological charge +1 or -1 , to excite the $K_{1}$ or $K_{2}$ state, respectively. To demonstrate that, we put such chiral source at the center of a triangle shaped ABN crystal with three surfaces oriented to the $\Gamma M$ directions. As shown in Fig. 2, the valley states $\left(K_{1}\right.$ and $\left.K_{2}\right)$ hosted by the $\mathrm{ABN}$ triangle are indeed excited. The sign of $\gamma$ determines whether a lattice site contains gain or loss. The attenuation-free scenario $(\gamma=0)$ predicts an almost entirely symmetric intensity profile between the $K_{1}$ and $K_{2}$ states. However, dependent on the sign of $\pm \gamma, K_{1,2}$ represent either an attenuating or an amplifying state. The intensities versus $\gamma$ for both the states
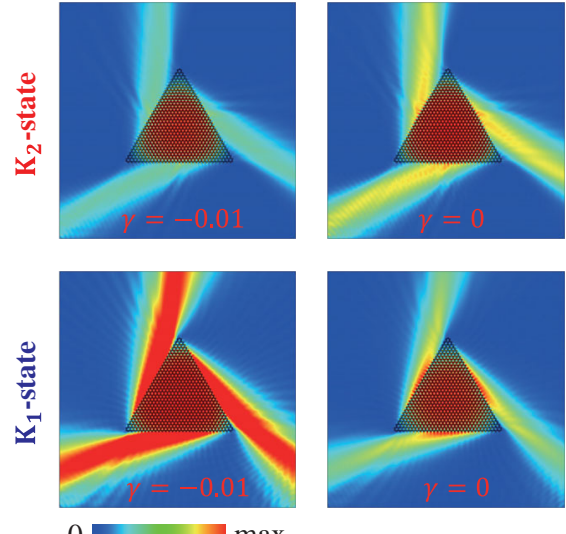
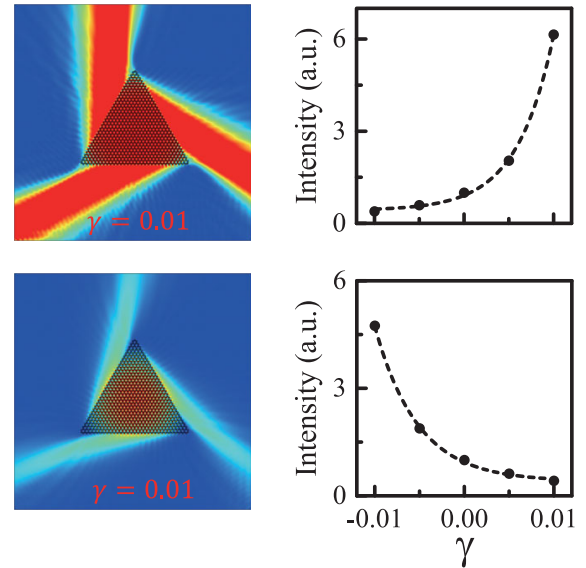

FIG. 2. The excitation of the valley states $K_{1}$ and $K_{2}$ by a chiral source of topological charge $m=1$ and $m=-1$, respectively, positioned in the center of the triangle-shaped sample for varying $\gamma$. The frequencies of the excitation source are the same as the real parts of the complex frequency of the states $K_{1}$ and $K_{2}$, respectively. Right panels: The black dots represent the intensity of the excited states, normalized by the intensity at $\gamma=0$. The black dashed lines are fitted to an exponential function. 
(a)

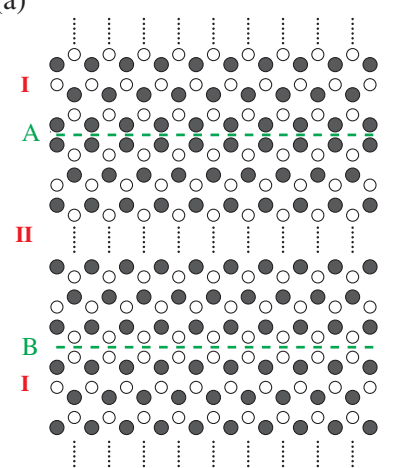

(b)

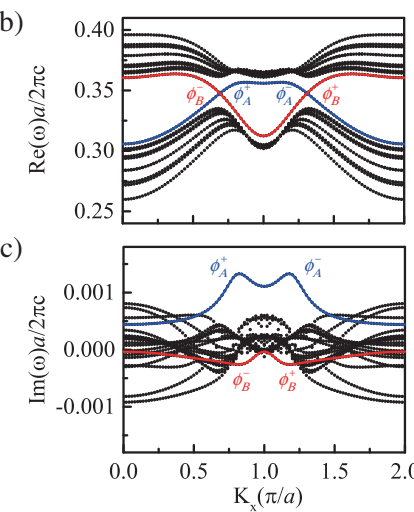

FIG. 3. (a) Heterostructure consisting of the original $A B N$ crystal in area I, and the mirror-symmetric ABN crystal in area II with two distinct horizontal interfaces. (b) and (c) The real and imaginary parts of the band diagram for the heterostructure shown in (a) with $\gamma=0.1$ are plotted. Blue and red solid lines indicate the VPESs on the upper and lower interfaces, respectively.

are given in the right panels of Fig. 2, which fit the exponential function as expected.

If we interchange cylinders $A$ and $B$ in the artificial ABN structure, we get a new system that is mirror symmetric to the original one with reference to the horizontal plane through point $P$ [see Fig. 1(a)]. This mirror symmetric ABN structure has exactly the same band diagram as the original one but the band gap is inverted. That is to say, in the new ABN crystal, the $K_{1}$ state carries a vortex of charge -1 while the $K_{2}$ state carries a vortex of charge +1 , the valley states get inverted compared to the original $\mathrm{ABN}$ crystal. However, what prevails in this scenario is that $K_{1}$ still is an attenuating state while the $K_{2}$ state remains responsible for wave amplification.

As it has been demonstrated [38], the VPESs can exist at the interface between two phononic crystals of inverted bands. Naturally, we could expect the existence of the VPESs along the interface of the original and the new $A B N$ crystal. But what is the effect of non-Hermiticity, and what influence does the introduction of gain and loss have on the edge states? To answer these questions, we consider a heterostructure consisting of the original $\mathrm{ABN}$ crystal in

area $\mathrm{I}$ and the new ABN crystal in area II, as shown in Fig. 3(a). By applying periodic boundary conditions on both the horizontal and the vertical interface (with the period of 46 layers in I and 42 layers in II), and calculating the dispersion relation along the $x$ direction for the structure with $\gamma=0.1$, the edge states at both the $A$ and $B$ interfaces (i.e., $\Gamma K$ direction of the $\mathrm{ABN}$ lattice) can be obtained simultaneously, as shown in Figs. 3(b) and 3(c), where (b) and (c), respectively, depict the real and imaginary parts of the band structure. Blue lines show the dispersion of the non-Hermitian VPESs along the $A$ interface, while red lines represent the states along the $B$ interface. It can be seen from Fig. 3(b) that we obtain pairs of counterpropagating VPESs along each interface, locked, respectively, to the $K$ and $K^{\prime}$ valleys. In detail, at the $A$ interface, the VPESs, traveling along the $+x$ direction $\phi_{A}^{+}$are locked to valley $K$, while the VPESs, traveling along the $-x$ direction $\phi_{A}^{-}$are locked to $K^{\prime}$; however, at the $B$ interface, the VPESs, traveling along the $+x$ direction $\phi_{B}^{+}$are locked to $K^{\prime}$, while the VPESs, traveling along the $-x$ direction $\phi_{B}^{+}$are locked to $K$. This distinct selection rule shows how valleypolarized interface states are projected from a specific valley and into a given direction, beyond which the nonHermitian ingredients add an additional degree of freedom. If we take a close look at the imaginary parts of the dispersion curve as shown in Fig. 3(c), we immediately see, for the present case with $\gamma=0.1$, that the VPESs along the $A$ interface, with positive imaginary frequencies are attenuating modes, while those along the $B$ interface, with negative imaginary frequency, are amplifying modes. It is easy to imagine that if we change the sign to $\gamma=-0.1$, according to symmetry, the real parts of the VPES spectrum at both the interfaces would remain unchanged, but the imaginary frequencies of the VPESs along the two interfaces would swap. It means that for the case with $\gamma=-0.1$, the VPESs along the $A$ interface would become amplifying modes while those at the $B$ interface, attenuating modes.

In what follows, we focus on the observation of excited VPESs propagating along these non-Hermitian ABN interfaces. As the VPESs on the $A$ interface are antisymmetric and difficult to excite by a normally incident beam [38], here we solely exemplify the excitation of the symmetric VPESs
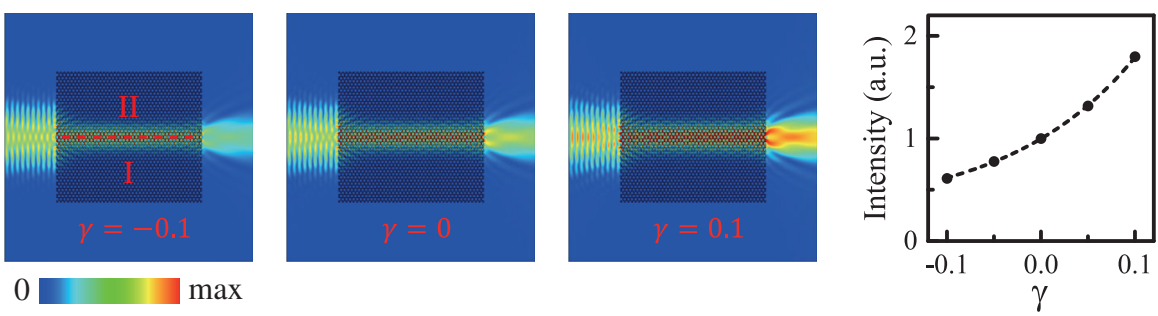

FIG. 4. Simulations of the acoustic fields of a Gaussian beam that is normally incident onto the sample consisting of ABN in area I, and mirror-symmetric ABN in area II, which are separated by a horizontal interface. Here the normalized frequency is 0.344 and we vary $\gamma$ from -0.1 to 0.1 . Right panel: normalized intensity versus $\gamma$, where the black dots from the simulations are fitted to an exponential function (dashed lines). 

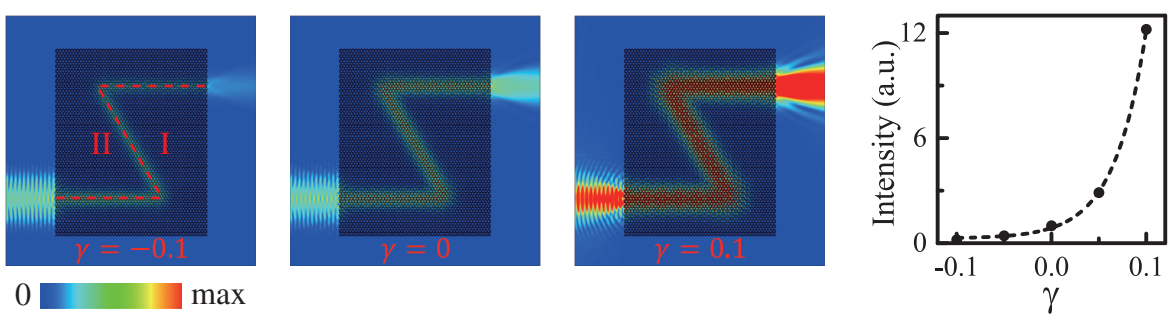

FIG. 5. Simulations of the acoustic fields of a Gaussian beam that is normally incident onto the sample consisting of ABN in area I, and mirror-symmetric $\mathrm{ABN}$ in area II, which are separated by a $Z$-shaped interface. Here the normalized frequency is 0.344 and we vary $\gamma$ from -0.1 to 0.1 . Right panel: normalized intensity versus $\gamma$, where the black dots from the simulations are fitted to an exponential function (dashed lines).

along the $B$ interface, as shown in Fig. 4 . We can see that, for various values of $\gamma$, the VPESs can always be excited, where for negative values of $\gamma$, the VPESs are attenuating, while for positive values, the VPESs start to amplify along the interface in full consistency to the above discussions.

The VPESs along the interface between two $A B N$ structures of inverted bands are unique: on the one hand, they are valley locked as has been mentioned, and on the other hand, they have the one-way propagation feature based on the absence of intervalley scattering thanks to their large separation in momentum space. Therefore, these edge states are immune to lattice imperfections such as defect that may be placed along the path of propagation. To demonstrate the one-way propagation robustness of the non-Hermitian VPESs, we simulate a Gaussian beam that is normally incident onto the sample with a $Z$-shaped interface at various values of $\gamma$, as shown in Fig. 5. We see that the acoustic waves travel smoothly along the curved path without being scattered back around the two sharp corners $\left(60^{\circ}\right)$. But for negative values of $\gamma$, the intensity through the path attenuates because the mode for this particular case is a lossy one, while for positive values of $\gamma$, the intensity grows since the mode now turns to be an amplifying one. As shown in the right panel of Fig. 5, the normalized intensity shows an exponential growth with increasing value of $\gamma$. It indicates that either attenuation or amplification of the obstacle-immune VPESs can be engineered through adjusting the gain or loss factor $\gamma$ in non-Hermitian artificial ABN, for which PT symmetry is always broken [56].

In conclusion, we have studied valley states and valleyprojected edge states in non-Hermitian acoustic boron nitride. Depending on the gain and loss factor in the system, the valley states and valley-projected edge states can be adjusted to be either attenuating or amplifying, however, always in the absence of a PT symmetry-breaking transition. Our findings have the potential to pave the way for exploring intensity-controllable valley transport in various classical but non-Hermitian systems, which could push forward advanced wave control for sensing and communication applications.

J.C. acknowledges the support from the European Research Council (ERC) through the Starting Grant
No. 714577 PHONOMETA and from the MINECO through a Ramón y Cajal grant (Grant No. RYC-201517156). This work was supported by the National Basic Research Program of China (Grant No. 2015CB755500); National Natural Science Foundation of China (Grants No. 11774275, No. 11674250, No. 11534013, and 11547310); Natural Science Foundation of Hubei Province (Grant No. 2017CFA042). J. C. acknowledge stimulating discussions with Daniel Torrent and Victor Tribaldos for the use of the supercomputer Uranus.

* Corresponding author.

jchriste@inst.uc3m.es

Corresponding author.

zyliu@whu.edu.cn

[1] O. Gunawan, Y. P. Shkolnikov, K. Vakili, T. Gokmen, E. P. De Poortere, and M. Shayegan, Valley Susceptibility of an Interacting Two-Dimensional Electron System, Phys. Rev. Lett. 97, 186404 (2006).

[2] K. Takashina, Y. Ono, A. Fujiwara, Y. Takahashi, and Y. Hirayama, Valley Polarization in $\mathrm{Si}(100)$ at Zero Magnetic Field, Phys. Rev. Lett. 96, 236801 (2006).

[3] Y. P. Shkolnikov, E. P. De Poortere, E. Tutuc, and M. Shayegan, Valley Splitting of AlAs Two-Dimensional Electrons in a Perpendicular Magnetic Field, Phys. Rev. Lett. 89, 226805 (2002).

[4] N. C. Bishop, M. Padmanabhan, K. Vakili, Y. P. Shkolnikov, E. P. De Poortere, and M. Shayegan, Valley Polarization and Susceptibility of Composite Fermions around a Filling Factor $\nu=\frac{3}{2}$, Phys. Rev. Lett. 98, 266404 (2007).

[5] Z. Zhu, A. Collaudin, B. Fauque, W. Kang, and K. Behnia, Field-induced polarization of Dirac valleys in bismuth, Nat. Phys. 8, 89 (2012).

[6] D. MacNeill, C. Heikes, K. F. Mak, Z. Anderson, A. Kormányos, V. Zólyomi, J. Park, and D. C. Ralph, Breaking of Valley Degeneracy by Magnetic Field in Monolayer $\mathrm{MoSe}_{2}$, Phys. Rev. Lett. 114, 037401 (2015).

[7] K. F. Mak, K. He, J. Shan, and T. F. Heinz, Control of valley polarization in monolayer $\mathrm{MoS}_{2}$ by optical helicity, Nat. Nanotechnol. 7, 494 (2012).

[8] H. Zeng, J. Dai, W. Yao, D. Xiao, and X. Cui, Valley polarization in $\mathrm{MoS}_{2}$ monolayers by optical pumping, Nat. Nanotechnol. 7, 490 (2012). 
[9] T. Cao et al., Valley-selective circular dichroism of monolayer molybdenum disulphide, Nat. Commun. 3, 887 (2012).

[10] A. Rycerz, J. Tworzydlo, and C. W. J. Beenakker, Valley filter and valley valve in graphene, Nat. Phys. 3, 172 (2007).

[11] Z. Wu, F. Zhai, F. M. Peeters, H. Q. Xu, and K. Chang, Valley-Dependent Brewster Angles and Goos-Hänchen Effect in Strained Graphene, Phys. Rev. Lett. 106, 176802 (2011).

[12] J. L. Garcia-Pomar, A. Cortija, and M. Nieto-Vesperinas, Fully Valley-Polarized Electron Beams in Graphene, Phys. Rev. Lett. 100, 236801 (2008).

[13] G.-B. Liu, D. Xiao, Y. Yao, X. Xu, and W. Yao, Electronic structures and theoretical modelling of two-dimensional group-VIB transition metal dichalcogenides, Chem. Soc. Rev. 44, 2643 (2015).

[14] D. Xiao, W. Yao, and Q. Niu, Valley-Contrasting Physics in Graphene: Magnetic Moment and Topological Transport, Phys. Rev. Lett. 99, 236809 (2007).

[15] W. Yao, D. Xiao, and Q. Niu, Valley-dependent optoelectronics from inversion symmetry breaking, Phys. Rev. B 77, 235406 (2008).

[16] D. Xiao, G. B. Liu, W. Feng, X. Xu, and W. Yao, Coupled Spin and Valley Physics in Monolayers of $\mathrm{MoS}_{2}$ and Other Group-VI Dichalcogenides, Phys. Rev. Lett. 108, 196802 (2012).

[17] K. F. Mak, K. L. McGill, J. Park, and P. L. McEuen, The valley Hall effect in $\mathrm{MoS}_{2}$ transistors, Science 344, 1489 (2014).

[18] G.-B. Liu, D. Xiao, Y. Yao, X. Xu, and W. Yao, Electronic structures and theoretical modelling of two-dimensional group-VIB transition metal dichalcogenides, Chem. Soc. Rev. 44, 2643 (2015).

[19] J. Lu, C. Qiu, M. Ke, and Z. Liu, Valley Vortex States in Sonic Crystals, Phys. Rev. Lett. 116, 093901 (2016).

[20] L. Ye, C. Qiu, J. Lu, X. Wen, Y. Shen, M. Ke, F. Zhang, and Z. Liu, Observation of acoustic valley vortex states and valley-chirality locked beam splitting, Phys. Rev. B 95, 174106 (2017).

[21] J.-W. Dong, X.-D. Chen, H. Zhu, Y. Wang, and X. Zhang, Valley photonic crystals for control of spin and topology, Nat. Mater. 16, 298 (2017).

[22] F. D. M. Haldane and S. Raghu, Possible Realization of Directional Optical Waveguides in Photonic Crystals with Broken Time-Reversal Symmetry, Phys. Rev. Lett. 100, 013904 (2008).

[23] Z. Wang, Y. D. Chong, J. D. Joannopoulos, and M. Soljačić, Reflection-Free One-Way Edge Modes in a Gyromagnetic Photonic Crystal, Phys. Rev. Lett. 100, 013905 (2008).

[24] Z. Wang, Y. Chong, J. D. Joannopoulos, and M. Soljačić, Observation of unidirectional backscattering-immune topological electromagnetic states, Nature (London) 461, 772 (2009).

[25] Y. Poo, R. X. Wu, Z. Lin, Y. Yang, and C. T. Chan, Experimental Realization of Self-Guiding Unidirectional Electromagnetic Edge States, Phys. Rev. Lett. 106, 093903 (2011).

[26] M. C. Rechtsman, J. M. Zeuner, Y. Plotnik, Y. Lumer, D. Podolsky, F. Dreisow, S. Nolte, M. Segev, and A. Szameit,
Photonic Floquet topological insulators, Nature (London) 496, 196 (2013).

[27] A. B. Khanikaev, S. Hossein Mousavi, W.-K. Tse, M. Kargarian, A. H. MacDonald, and G. Shvets, Photonic topological insulators, Nat. Mater. 12, 233 (2013).

[28] W. Chen, S.-J. Jiang, X.-D. Chen, B. Zhu, L. Zhou, J.-W. Dong, and C. T. Chan, Experimental realization of photonic topological insulator in a uniaxial metacrystal waveguide, Nat. Commun. 5, 5782 (2014).

[29] L. Lu, J. D. Joannopoulos, and M. Soljačić, Topological photonics, Nat. Photonics 8, 821 (2014).

[30] T. Ma, A. B. Khanikaev, S. H. Mousavi, and G. Shvets, Guiding Electromagnetic Waves around Sharp Corners: Topologically Protected Photonic Transport in Metawaveguides, Phys. Rev. Lett. 114, 127401 (2015).

[31] L. H. Wu and X. Hu, Scheme for Achieving a Topological Photonic Crystal by Using Dielectric Material, Phys. Rev. Lett. 114, 223901 (2015).

[32] X. Ni, C. He, X.-C. Sun, X.-p. Liu, M.-H. Lu, L. Feng, and Y.-F. Chen, Topologically protected one-way edge mode in networks of acoustic resonators with circulating air flow, New J. Phys. 17, 053016 (2015).

[33] Z. Yang, F. Gao, X. Shi, X. Lin, Z. Gao, Y. Chong, and B. Zhang, Topological Acoustics, Phys. Rev. Lett. 114, 114301 (2015).

[34] P. Wang, L. Lu, and K. Bertoldi, Topological Phononic Crystals with One-Way Elastic Edge Waves, Phys. Rev. Lett. 115, 104302 (2015).

[35] M. Xiao, G. Ma, Z. Yang, Z. Q. Zhang, and C. T. Chan, Geometric phase and band inversion in periodic acoustic systems, Nat. Phys. 11, 240 (2015).

[36] M. Xiao, W. Chen, W. He, and C. T. Chan, Synthetic gauge flux and Weyl points in acoustic systems, Nat. Phys. 11, 920 (2015).

[37] V. Peano, C. Brendel, M. Schmidt, and F. Marquardt, Topological Phases of Sound and Light, Phys. Rev. X 5, 031011 (2015).

[38] J. Lu, C. Qiu, L. Ye, X. Fan, M. Ke, F. Zhang, and Z. Liu, Observation of topological valley transport of sound in sonic crystals, Nat. Phys. 13, 369 (2017).

[39] C. M. Bender, D. C. Brody, and H. F. Jones, Complex Extension of Quantum Mechanics, Phys. Rev. Lett. 89, 270401 (2002).

[40] C. M. Bender, D. C. Brody, and H. F. Jones, Extension of PT-symmetric quantum mechanics to quantum field theory with cubic interaction, Phys. Rev. D 70, 025001 (2004).

[41] I. Y. Goldsheid and B. A. Khoruzhenko, Distribution of Eigenvalues in Non-Hermitian Anderson Models, Phys. Rev. Lett. 80, 2897 (1998).

[42] C. Dembowski, B. Dietz, H.-D. Gräf, H. L. Harney, A. Heine, W. D. Heiss, and A. Richter, Observation of a Chiral State in a Microwave Cavity, Phys. Rev. Lett. 90, 034101 (2003).

[43] K. G. Makris, R. El-Ganainy, D. N. Christodoulides, and Ziad H. Musslimani, Beam Dynamics in PT Symmetric Optical Lattices, Phys. Rev. Lett. 100, 103904 (2008).

[44] Y. D. Chong, L. Ge, H. Cao, and A. D. Stone, Coherent Perfect Absorbers: Time-Reversed Lasers, Phys. Rev. Lett. 105, 053901 (2010). 
[45] L. Ge, Y. D. Chong, and A. D. Stone, Conservation relations and anisotropic transmission resonances in one-dimensional PT-symmetric photonic heterostructures, Phys. Rev. A 85, 023802 (2012).

[46] Y. Sun, W. Tan, H. Q. Li, J. Li, and H. Chen, Experimental Demonstration of a Coherent Perfect Absorber with PT Phase Transition, Phys. Rev. Lett. 112, 143903 (2014).

[47] C.E. Ruter, K. G. Makris, R. El-Ganainy, D. N. Christodoulides, M. Segev, and D. Kip, Observation of parity-time symmetry in optics, Nat. Phys. 6, 192 (2010).

[48] A. Szameit, M. C. Rechtsman, O. Bahat-Treidel, and M. Segev, PT-symmetry in honeycomb photonic lattices, Phys. Rev. A 84, 021806 (2011).

[49] H. Ramezani, T Kottos, V. Kovanis, and D. N. Christodoulides, Exceptional-point dynamics in photonic honeycomb lattices with PT symmetry, Phys. Rev. A 85, 013818 (2012).

[50] B.-I. Popa and S. A. Cummer, Non-reciprocal and highly nonlinear active acoustic metamaterials, Nat. Commun. 5, 3398 (2014).

[51] J. Christensen, M. Willatzen, V. R. Velasco, and M.-H. Lu, Parity-Time Synthetic Phononic Media, Phys. Rev. Lett. 116, 207601 (2016).
[52] R. Fleury, D. L. Sounas, and A. Alu, An invisible acoustic sensor based on parity-time symmetry, Nat. Commun. 6, 5905 (2015).

[53] Y. Aurégan and V. Pagneux, PT-Symmetric Scattering in Flow Duct Acoustics, Phys. Rev. Lett. 118, 174301 (2017).

[54] Y. Li, C. Shen, Y. Xie, J. Li, W. Wang, S. A. Cummer, and Y. Jing, Tunable Asymmetric Transmission via Lossy Acoustic Metasurfaces, Phys. Rev. Lett. 119, 035501 (2017).

[55] T. Liu, X. Zhu, F. Chen, S. Liang, and J. Zhu, Unidirectional Wave Vector Manipulation in Two-Dimensional Space with an All Passive Acoustic Parity-Time-Symmetric Metamaterials Crystal, Phys. Rev. Lett. 120, 124502 (2018).

[56] See Supplemental Material at http://link.aps.org/ supplemental/10.1103/PhysRevLett.120.246601, for nonHermitian ABN with complex bulk moduli, $P T$ symmetry phase diagram, and practical realizations, which includes Ref. [57].

[57] Z. Hou and B. Assouar, Tunable elastic parity-time symmetric structure based on the shunted piezoelectric materials, J. Appl. Phys. 123, 085101 (2018).

[58] J. O. Vasseur, B. Djafari-Rouhani, L. Dobrzynski, and P. A. Deymier, Acoustic band gaps in fibre composite materials of boron nitride structure, J. Phys. Condens. Matter 9, 7327 (1997). 